\title{
EAl EndorsedTransactions

\section{An Experimental Study with Tensor Flow for Characteristic mining of Mathematical Formulae from a Document}

\author{
K. N. Brahmaji Rao ${ }^{1, *}$, G. Srinivas ${ }^{2}$ and P. V. G. D. Prasad Reddy ${ }^{1}$ \\ ${ }^{1}$ Andhra University, Visakhapatnam, Andhra Pradesh, India \\ ${ }^{2}$ ANITS, Visakhapatnam, Andhra Pradesh, India
}

\section{Abstract}

Through this article a deep learning technique is proposed for the extraction and classification of mathematical keywords from textual documents. Extraction of math keywords from textual data is predominant problem as textual documents contain a culmination of mathematical symbols and literals from natural language such as alphabets and words. Separation of these textual words embedded in the mathematical formulae is a complex task. Our proposed technique solves this critical problem of extracting mathematical keywords from textual documents using techniques such as stemming, tokenization and clustering mathematical keywords based on a training set of mathematical keyword and formulae pairs. The performance of the proposed technique is measured using the metrics such as retrieval time, Sensitivity, Accuracy, FPR, FNR, and FDR are used for appraisal of the proposed technique.

Received on 17 January 2019, accepted on 20 May 2019, published on 10 June 2019

Copyright (C) 2019 K. N. Brahmaji Rao et al., licensed to EAI. This is an open access article distributed under the terms of the Creative Commons Attribution licence (http://creativecommons.org/licenses/by/3.0/), which permits unlimited use, distribution and reproduction in any medium so long as the original work is properly cited.

doi: 10.4108/eai.10-6-2019.159097

*Corresponding author. Email:brahmaji77@gmail.com

\section{Introduction}

TensorFlow is used for the high performance of numerical computation. It is an open source software library. It is flexible for simple deployment of computation across different platforms. It was developed by Google Brain team within Google's AI organization. It is flexible for numerical computations used in many scientific fields and gives strong support for machine learning and deep learning.

Text can be classified as well as clustered by using Tensorflow. The chief advantage of Tensorflow is that it is a base documentation that can be used to generate Deep
Learning models directly. The text classification with Tensorflow will be separated into numerous segments.

The first segment deals the text pre-processing and formation of the container of words. Second segment trains the text classifier and finally performs the testing using the classifier $[1,2,3,4,5,6,7,8]$.

\subsection{Stemming}

The procedure applied to a single word to obtain its root is called stemming. The words that are used in a sentence are often derived. To normalize our procedure, we would like to trunk such words and end up with only root words [4]. 
For example, after stemming following words "writing", "written", and "writer" ends up with their root word "write".

\subsection{Tokenization}

The words in a sentence are called Tokens. Tokenization is a process of finding unique words in the text from a given piece of text.

Tokenization splits the sentence " $\mathrm{C}$ Programming Language" in to a set of token list ["C", "Programming", and "Language"] [4].

\subsection{Bag of Words}

The Bag of Words is the process of generating an exclusive list of words. It acts as a tool for characteristic generation.

\section{Steps involved in the retrieval of Math formulae with TensorFlow}

\subsection{Training the Data}

After the preparation of data, we have to train the model. In the proposed approach, we first take a CSV file which is a sample data. In the first column the file contains the entire formula notation and the second column contains related text for that. Likewise, we have to prepare huge data sample. After preparing the huge data sample the CSV file need to be converted in to JSON File by importing required python libraries [4].

\subsection{Loading and Pre-processing of Data}

In this step, we load the attained JSON data that we have created for training. Let us presume that we have that json data stored in a file named "testdata.json". After loading the data, we would have to perform some required operations called pre-processing for cleaning the data like elimination of bag of words, tokenizing, stemming etc.

The exclusive stemmed words in all the sentences provided for training are placed in one list. The other list clutches the different categories. The "docs" list is the output of this step which includes the words from each sentence and which category the sentence fit in. The document is (["limit", "x to 0", "y to 0"], "sigma") is an example [4].

\subsection{Convert the processed data to Tensorflow requirements and instigate Tensorflow text categorization}

After the above two steps the documents are in text form, a bag of words to be applied in order to translate the sentence in to numeric array. As Tensorflow being a math library accepts the data in the numeric form. A deep Neural Network is developed and used for the training of the proposed model. Now the categorization of Tensorflow text document is performed on documents in the right form [4].

\subsection{Assessment of the Tensorflow Categorization Model}

After the completion of training, the text file should be loaded into our program and then parse every line in the text file with our neural network training model to check with how much accuracy the model retrieves math formulae.

During training, the model was able to correctly classify all the sentences. The accuracy and efficiency of retrieval depends on the size of the training document. For example primarily we train our model with 25 lines of text data and the testing is performed with a document contains 10 lines of text file and the accuracy is around $98 \%$. In this model we also calculated the time for performing complete program and for the above document with 10 lines of text its takes around 18-20 milliseconds. Depending on the size of text sample the time and accuracy will increase [4].

CSV to JSON conversion

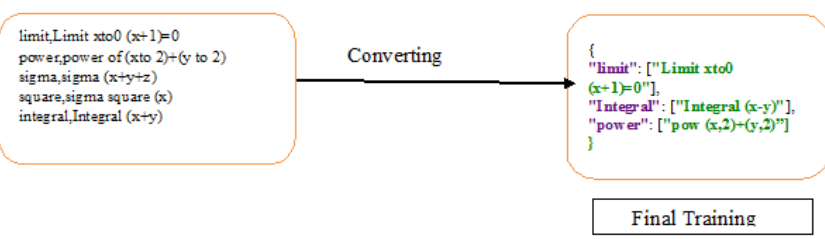

Figure 1. Procedure of TensorFlow based retrieval of mathematical formulae. 


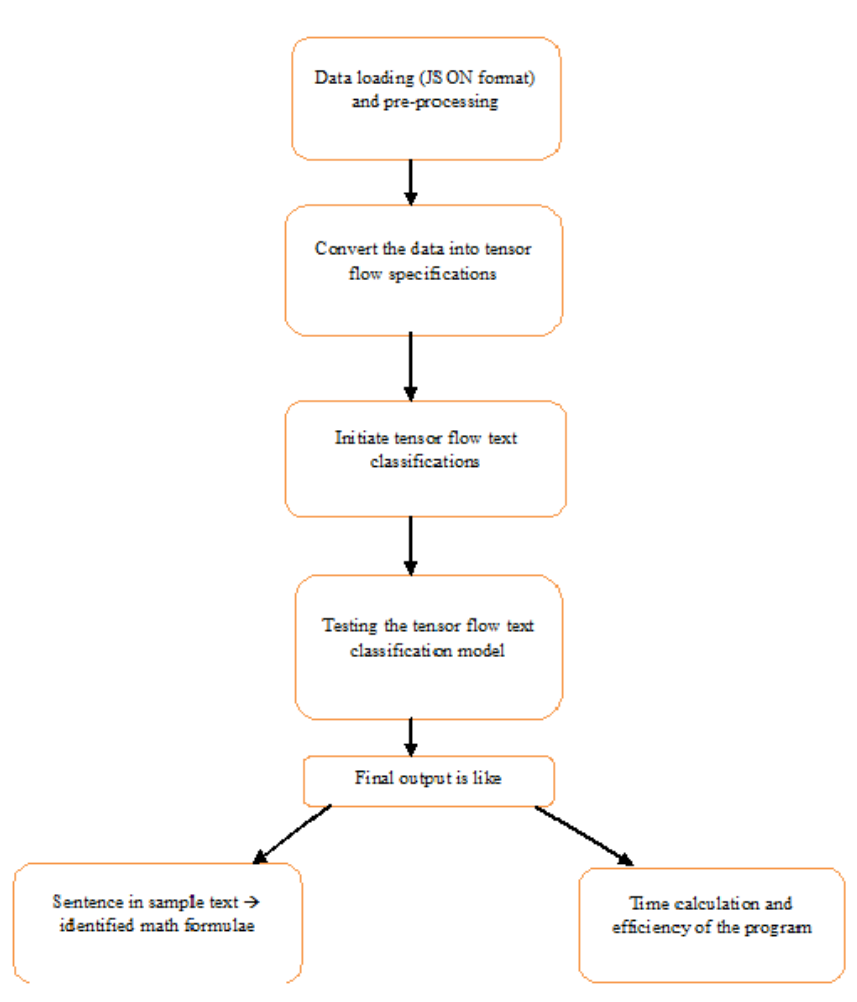

Figure 2. Training model and testing with text file

\section{Experimental Analysis and Results}

For calculating the efficiency of the text document first the JSON file should be prepared for training. The JSON file is prepared from CSV file which contains more than 120 different formulae. Now the JSON format is loaded into our program to train the data. After training the data, some sample text files are loaded for checking the efficiency of program based on how the training sample identifies formulae in the text file. The efficiency of various sizes of test documents are matched with training document and the results are tabulated in tables $1-3[9,10$, $11,12,13,14,15,16,17]$.

\subsection{Efficiency}

Efficiency is measured as the number of formulae retrieved from the number of the number of formulae in the training document. The efficiency of the proposed math formulae retrieval system depends on the size of the training data. The efficiency is increased with increased number of math data in the training document.

Efficiency $=$ (number of lines identified correctly/total number of lines)*100

Table 1. The above tabulated value represents overall Efficiency Measure with Tensorflow from a document of 20 samples.

\begin{tabular}{|l|l|l|}
\hline $\begin{array}{l}20 \text { Number of } \\
\text { testing formulae }\end{array}$ & $\begin{array}{l}\text { Number of } \\
\text { formulas } \\
\text { retrieved }\end{array}$ & Total efficiency \\
\hline Addition & 19 & $95 \%$ \\
\hline Combination & 19 & $95 \%$ \\
\hline Differentiation & 20 & $100 \%$ \\
\hline Exponential & 18 & $90 \%$ \\
\hline Factorial & 19 & $95 \%$ \\
\hline Integral & 19 & $95 \%$ \\
\hline Limit & 20 & $100 \%$ \\
\hline Permutation & 18 & $90 \%$ \\
\hline Sigma & 16 & $80 \%$ \\
\hline Square root & 16 & $80 \%$ \\
\hline Square & 16 & $80 \%$ \\
\hline Trigonometric & 19 & $95 \%$ \\
\hline
\end{tabular}

Table 2. The above tabulated value represents Overall Efficiency Measure with Tensorflow from a document of 40 samples.

\begin{tabular}{|l|l|l|}
\hline $\begin{array}{l}40 \text { Number of } \\
\text { testing formulae }\end{array}$ & $\begin{array}{l}\text { Number of } \\
\text { formulas } \\
\text { retrieved }\end{array}$ & Total efficiency \\
\hline Addition & 38 & $95 \%$ \\
\hline Combination & 39 & $97.5 \%$ \\
\hline Differentiation & 40 & $100 \%$ \\
\hline Exponential & 38 & $95 \%$ \\
\hline Factorial & 39 & $97.5 \%$ \\
\hline Integral & 39 & $97.5 \%$ \\
\hline Limit & 40 & $100 \%$ \\
\hline Permutation & 36 & $90 \%$ \\
\hline Sigma & 34 & $85 \%$ \\
\hline Square root & 36 & $90 \%$ \\
\hline Square & 34 & $85 \%$ \\
\hline Trigonometric & 38 & $95 \%$ \\
\hline
\end{tabular}

Table 3. The above tabulated value represents overall Efficiency Measure with Tensorflow from a document of 60 samples. 


\begin{tabular}{|l|l|l|}
\hline $\begin{array}{l}\text { 60 Number of } \\
\text { testing formulae }\end{array}$ & $\begin{array}{l}\text { Number of } \\
\text { formulas } \\
\text { retrieved }\end{array}$ & Total efficiency \\
\hline Addition & 58 & $96.7 \%$ \\
\hline Combination & 60 & $100 \%$ \\
\hline Differentiation & 60 & $100 \%$ \\
\hline Exponential & 59 & $98.3 \%$ \\
\hline Factorial & 59 & $98.3 \%$ \\
\hline Integral & 58 & $96.7 \%$ \\
\hline Limit & 60 & $100 \%$ \\
\hline Permutation & 58 & $96.7 \%$ \\
\hline Sigma & 56 & $100 \%$ \\
\hline Square root & 58 & $100 \%$ \\
\hline Square & 57 & $95 \%$ \\
\hline Trigonometric & 59 & $98.3 \%$ \\
\hline
\end{tabular}

\begin{tabular}{|l|l|l|c|c|}
\hline & & $\mathrm{ms}$ & & \\
\hline Integral & 19 & $\begin{array}{l}63.20 \\
\mathrm{~ms}\end{array}$ & $0.30 \mathrm{~ms}$ & $63.50 \mathrm{~ms}$ \\
\hline Limit & 20 & $\begin{array}{l}61.80 \\
\mathrm{~ms}\end{array}$ & $0.09 \mathrm{~ms}$ & $61.89 \mathrm{~ms}$ \\
\hline Permutation & 18 & $\begin{array}{l}62.42 \\
\mathrm{~ms}\end{array}$ & $0.06 \mathrm{~ms}$ & $62.48 \mathrm{~ms}$ \\
\hline Sigma & 16 & $\begin{array}{l}51.97 \\
\mathrm{~ms}\end{array}$ & $0.13 \mathrm{~ms}$ & $52.10 \mathrm{~ms}$ \\
\hline Square root & 16 & $\begin{array}{l}58.54 \\
\mathrm{~ms}\end{array}$ & $0.11 \mathrm{~ms}$ & $58.65 \mathrm{~ms}$ \\
\hline Square & 16 & $\begin{array}{l}58.40 \\
\mathrm{~ms}\end{array}$ & $0.10 \mathrm{~ms}$ & $58.50 \mathrm{~ms}$ \\
\hline Trigonometric & 19 & $\begin{array}{l}51.07 \\
\mathrm{~ms}\end{array}$ & $0.01 \mathrm{~ms}$ & $51.08 \mathrm{~ms}$ \\
\hline
\end{tabular}

From the above tables 1-3 the efficiency of math formulae retrieval with Tesorflow is measured and from the table it is concluded that the efficiency is more if number of samples increases. i.e. for example out of 20 lines of text file if the program identifies around 19 lines then efficiency of proposed model is $95 \%$. The efficiency always depends on the size of training sample.

\subsection{Time Analysis}

We conducted quite a few experiments to calculate the time taken for preparing the training model and for testing with various sizes of sample text files. In our experimentation we prepared the training model with over 120 lines of text in JSON format. Now the sample text files contains more than 20 lines are tested with the training document and the output of the program obtained within 40-60 milliseconds of time with high accuracy. Note the time may vary from one computer to another depending upon the ram and computer specifications the time calculations for various sizes of testing formulae are accessible in table 4-6 [9, 10, 11] From the above tables 3-6 it is clear that the training time and testing time required gradually decreases as the number of formulae increases.

Table 4. The above tabulated value represents time taken for retrieving matched formulae with

Tensorflow from a document of 20 samples along with testing time and training time.

\begin{tabular}{|l|l|l|l|l|}
\hline $\begin{array}{l}20 \text { Number of } \\
\text { testing } \\
\text { formulae }\end{array}$ & $\begin{array}{l}\text { Number } \\
\text { of } \\
\text { formulas } \\
\text { retrieved }\end{array}$ & $\begin{array}{l}\text { Training } \\
\text { Time in } \\
\mathrm{ms}\end{array}$ & $\begin{array}{l}\text { Testing } \\
\text { Time in } \\
\mathrm{ms}\end{array}$ & $\begin{array}{l}\text { Total } \\
\text { Time in } \\
\mathrm{ms}\end{array}$ \\
\hline Addition & 19 & $41.00 \mathrm{~ms}$ & $1.33 \mathrm{~ms}$ & $42.33 \mathrm{~ms}$ \\
\hline Combination & 19 & $41.00 \mathrm{~ms}$ & $0.43 \mathrm{~ms}$ & $41.43 \mathrm{~ms}$ \\
\hline Differentiation & 20 & $41.00 \mathrm{~ms}$ & $0.48 \mathrm{~ms}$ & $41.48 \mathrm{~ms}$ \\
\hline Exponential & 18 & $\begin{array}{l}60.00 \\
\mathrm{~ms}\end{array}$ & $1.23 \mathrm{~ms}$ & $61.23 \mathrm{~ms}$ \\
\hline Factorial & 19 & 60.00 & $0.90 \mathrm{~ms}$ & $60.90 \mathrm{~ms}$ \\
\hline
\end{tabular}

Table 5. The above tabulated value represents time taken for retrieving matched formulae with

Tensorflow from a document of 40 samples along with testing time and training time.

\begin{tabular}{|l|l|l|l|l|}
\hline $\begin{array}{l}40 \text { Number of } \\
\text { testing } \\
\text { formulae }\end{array}$ & $\begin{array}{l}\text { Number } \\
\text { of } \\
\text { formulas } \\
\text { retrieved }\end{array}$ & $\begin{array}{l}\text { Training } \\
\text { Time in } \\
\mathrm{ms}\end{array}$ & $\begin{array}{l}\text { Testing } \\
\text { Time in } \\
\mathrm{ms}\end{array}$ & $\begin{array}{l}\text { Total } \\
\text { Time in } \\
\mathrm{ms}\end{array}$ \\
\hline Addition & 38 & $43.00 \mathrm{~ms}$ & $2.65 \mathrm{~ms}$ & $45.65 \mathrm{~ms}$ \\
\hline Combination & 39 & $41.00 \mathrm{~ms}$ & $2.36 \mathrm{~ms}$ & $43.36 \mathrm{~ms}$ \\
\hline Differentiation & 40 & $43.00 \mathrm{~ms}$ & $1.48 \mathrm{~ms}$ & $44.48 \mathrm{~ms}$ \\
\hline Exponential & 38 & $\begin{array}{l}64.00 \\
\mathrm{~ms}\end{array}$ & $1.23 \mathrm{~ms}$ & $65.23 \mathrm{~ms}$ \\
\hline Factorial & 39 & $\begin{array}{l}63.00 \\
\mathrm{~ms}\end{array}$ & $1.00 \mathrm{~ms}$ & $64.00 \mathrm{~ms}$ \\
\hline Integral & 39 & $\begin{array}{l}65.20 \\
\mathrm{~ms}\end{array}$ & $0.32 \mathrm{~ms}$ & $65.52 \mathrm{~ms}$ \\
\hline Limit & 40 & $\begin{array}{l}63.80 \\
\mathrm{~ms}\end{array}$ & $0.12 \mathrm{~ms}$ & $63.92 \mathrm{~ms}$ \\
\hline Permutation & 36 & $\begin{array}{l}65.42 \\
\mathrm{~ms}\end{array}$ & $0.16 \mathrm{~ms}$ & $65.58 \mathrm{~ms}$ \\
\hline Sigma & 34 & $\begin{array}{l}55.86 \\
\mathrm{~ms}\end{array}$ & $0.23 \mathrm{~ms}$ & $56.09 \mathrm{~ms}$ \\
\hline Square root & 36 & $\begin{array}{l}60.64 \\
\mathrm{~ms}\end{array}$ & $0.31 \mathrm{~ms}$ & $60.95 \mathrm{~ms}$ \\
\hline Square & 34 & $\begin{array}{l}61.62 \\
\mathrm{~ms}\end{array}$ & $0.30 \mathrm{~ms}$ & $61.92 \mathrm{~ms}$ \\
\hline Trigonometric & 38 & $\begin{array}{l}55.27 \\
\mathrm{~ms}\end{array}$ & $0.10 \mathrm{~ms}$ & $55.37 \mathrm{~ms}$ \\
\hline
\end{tabular}

Table 6 . The above tabulated value represents time taken for retrieving matched formulae with

Tensorflow from a document of 60 samples along with testing time and training time.

\begin{tabular}{|l|l|l|l|l|}
\hline $\begin{array}{l}60 \text { Number of } \\
\text { testing } \\
\text { formulae }\end{array}$ & $\begin{array}{l}\text { Number } \\
\text { of } \\
\text { formulas } \\
\text { retrieved }\end{array}$ & $\begin{array}{l}\text { Training } \\
\text { Time in } \\
\mathrm{ms}\end{array}$ & $\begin{array}{l}\text { Testing } \\
\text { Time in } \\
\mathrm{ms}\end{array}$ & $\begin{array}{l}\text { Total } \\
\text { Time in } \\
\mathrm{ms}\end{array}$ \\
\hline Addition & 58 & $43.05 \mathrm{~ms}$ & $2.79 \mathrm{~ms}$ & $45.84 \mathrm{~ms}$ \\
\hline
\end{tabular}




\begin{tabular}{|l|l|l|l|l|}
\hline Combination & 60 & $41.15 \mathrm{~ms}$ & $2.41 \mathrm{~ms}$ & $43.56 \mathrm{~ms}$ \\
\hline Differentiation & 60 & $43.22 \mathrm{~ms}$ & $1.61 \mathrm{~ms}$ & $44.83 \mathrm{~ms}$ \\
\hline Exponential & 59 & $64.34 \mathrm{~ms}$ & $1.35 \mathrm{~ms}$ & $65.69 \mathrm{~ms}$ \\
\hline Factorial & 59 & $63.41 \mathrm{~ms}$ & $1.12 \mathrm{~ms}$ & $64.53 \mathrm{~ms}$ \\
\hline Integral & 58 & $65.27 \mathrm{~ms}$ & $0.32 \mathrm{~ms}$ & $65.59 \mathrm{~ms}$ \\
\hline Limit & 60 & $64.01 \mathrm{~ms}$ & $0.12 \mathrm{~ms}$ & $64.13 \mathrm{~ms}$ \\
\hline Permutation & 58 & $66.16 \mathrm{~ms}$ & $0.16 \mathrm{~ms}$ & $66.32 \mathrm{~ms}$ \\
\hline Sigma & 56 & $56.01 \mathrm{~ms}$ & $0.23 \mathrm{~ms}$ & $56.24 \mathrm{~ms}$ \\
\hline Square root & 58 & $60.77 \mathrm{~ms}$ & $0.31 \mathrm{~ms}$ & $61.08 \mathrm{~ms}$ \\
\hline Square & 57 & $61.72 \mathrm{~ms}$ & $0.30 \mathrm{~ms}$ & $62.02 \mathrm{~ms}$ \\
\hline Trigonometric & 59 & $55.34 \mathrm{~ms}$ & $0.10 \mathrm{~ms}$ & $55.44 \mathrm{~ms}$ \\
\hline
\end{tabular}

\subsection{Sensitivity Measure}

Sensitivity is used to measure the ratio of actual math keywords that are exactly matched with the training document from the text file, supplied as an input. The overall Sensitivity Measure with Tensorflow model is presented in tables 7-9 and from the tables it is obvious that with huge training data more number of matched math formulae from the text document will be retrieved results high sensitivity.

Sensitivity can be expressed as:

$$
\text { Sensitivity }(\mathrm{S})=\frac{n(T p)}{n(T p)+n(F n)}
$$

Where,

$$
\begin{aligned}
& n(T p)=\text { Number of True Positives } \\
& n(F n)=\text { Number of False Negatives }
\end{aligned}
$$

Table 7. The above tabulated value represents overall Sensitivity Measure with Tensorflow from a document of 20 samples.

\begin{tabular}{|l|l|l|l|}
\hline $\begin{array}{l}\text { 20 Number of } \\
\text { testing formulae }\end{array}$ & $\boldsymbol{n}(\boldsymbol{T p})$ & $\boldsymbol{n}(\boldsymbol{F n})$ & Sensitivity \\
\hline Addition & 19 & 1 & $95 \%$ \\
\hline Combination & 19 & 1 & $95 \%$ \\
\hline Differentiation & 20 & 0 & $100 \%$ \\
\hline Exponential & 18 & 2 & $90 \%$ \\
\hline Factorial & 19 & 1 & $95 \%$ \\
\hline Integral & 19 & 1 & $95 \%$ \\
\hline Limit & 20 & 0 & $100 \%$ \\
\hline Permutation & 18 & 2 & $90 \%$ \\
\hline Sigma & 16 & 4 & $80 \%$ \\
\hline Square root & 16 & 4 & $80 \%$ \\
\hline Square & 16 & 4 & $80 \%$ \\
\hline Trigonometric & 19 & 1 & $95 \%$ \\
\hline
\end{tabular}

Table 8. The above tabulated value represents overall Sensitivity Measure with Tensorflow from a document of 40 samples.

\begin{tabular}{|l|l|l|l|}
\hline $\begin{array}{l}40 \text { Number of } \\
\text { testing formulae }\end{array}$ & $\boldsymbol{n}(\boldsymbol{T p})$ & $\boldsymbol{n}(\boldsymbol{F n})$ & Sensitivity \\
\hline Addition & 38 & 2 & $95 \%$ \\
\hline Combination & 39 & 1 & $97.5 \%$ \\
\hline Differentiation & 40 & 0 & $100 \%$ \\
\hline Exponential & 38 & 2 & $95 \%$ \\
\hline Factorial & 39 & 1 & $97.5 \%$ \\
\hline Integral & 39 & 1 & $97.5 \%$ \\
\hline Limit & 40 & 0 & $100 \%$ \\
\hline Permutation & 36 & 4 & $90 \%$ \\
\hline Sigma & 34 & 6 & $85 \%$ \\
\hline Square root & 36 & 4 & $90 \%$ \\
\hline Square & 34 & 6 & $85 \%$ \\
\hline Trigonometric & 38 & 2 & $95 \%$ \\
\hline
\end{tabular}

Table 9. The above tabulated value represents overall Sensitivity Measure with Tensorflow from a document of 60 samples.

\begin{tabular}{|l|l|l|l|}
\hline $\begin{array}{l}60 \text { Number of } \\
\text { testing formulae }\end{array}$ & $\boldsymbol{n}(\boldsymbol{T p})$ & $\boldsymbol{n}(\boldsymbol{F n})$ & Sensitivity \\
\hline Addition & 58 & 2 & $96.7 \%$ \\
\hline Combination & 60 & 0 & $100 \%$ \\
\hline Differentiation & 60 & 0 & $100 \%$ \\
\hline Exponential & 59 & 1 & $98.3 \%$ \\
\hline Factorial & 59 & 1 & $98.3 \%$ \\
\hline Integral & 58 & 2 & $96.7 \%$ \\
\hline Limit & 60 & 0 & $100 \%$ \\
\hline Permutation & 58 & 2 & $96.7 \%$ \\
\hline Sigma & 56 & 4 & $93.3 \%$ \\
\hline Square root & 58 & 2 & $96.7 \%$ \\
\hline Square & 57 & 3 & $95 \%$ \\
\hline Trigonometric & 59 & 1 & $98.3 \%$ \\
\hline
\end{tabular}

\subsection{False Negative Rate}

FNR is the number of Math formulae those responding negative on the test, means the formulae which are wrongly retrieved as obtainable in tables 10-12. The data in the tables illustrates that FNR value decrease with increase in the number of formulae in the text document.

FNR-False Negative Rate $=n\left(F_{n}\right) / n\left(F_{n}\right)+n\left(T_{p}\right)$

Table 10. The above tabulated value represents overall FNR Measure with Tensorflow from a document of 20 samples.

\begin{tabular}{|l|l|l|l|}
\hline $\begin{array}{l}\text { 20 Number of } \\
\text { testing formulae }\end{array}$ & $\boldsymbol{n}(\boldsymbol{T p})$ & $\boldsymbol{n}(\boldsymbol{F n})$ & $\begin{array}{l}\text { False Negative } \\
\text { Rate }\end{array}$ \\
\hline Addition & 19 & 1 & $5 \%$ \\
\hline Combination & 19 & 1 & $5 \%$ \\
\hline Differentiation & 20 & 0 & $0 \%$ \\
\hline Exponential & 18 & 2 & $10 \%$ \\
\hline Factorial & 19 & 1 & $5 \%$ \\
\hline
\end{tabular}




\begin{tabular}{|l|l|l|l|}
\hline Integral & 19 & 1 & $5 \%$ \\
\hline Limit & 20 & 0 & $0 \%$ \\
\hline Permutation & 18 & 2 & $10 \%$ \\
\hline Sigma & 16 & 4 & $20 \%$ \\
\hline Square root & 16 & 4 & $20 \%$ \\
\hline Square & 16 & 4 & $20 \%$ \\
\hline Trigonometric & 19 & 1 & $5 \%$
\end{tabular}

Table 11. The above tabulated value represents overall FNR Measure with Tensorflow from a document of 40 samples.

\begin{tabular}{|l|l|l|l|}
\hline $\begin{array}{l}40 \text { Number of } \\
\text { testing formulae }\end{array}$ & $\boldsymbol{n}(\boldsymbol{T p})$ & $\boldsymbol{n}(\boldsymbol{F n})$ & $\begin{array}{l}\text { False Negative } \\
\text { Rate }\end{array}$ \\
\hline Addition & 38 & 2 & $5 \%$ \\
\hline Combination & 39 & 1 & $2.5 \%$ \\
\hline Differentiation & 40 & 0 & $0 \%$ \\
\hline Exponential & 38 & 2 & $5 \%$ \\
\hline Factorial & 39 & 1 & $2.5 \%$ \\
\hline Integral & 39 & 1 & $2.55 \%$ \\
\hline Limit & 40 & 0 & $0 \%$ \\
\hline Permutation & 36 & 4 & $10 \%$ \\
\hline Sigma & 34 & 6 & $15 \%$ \\
\hline Square root & 36 & 4 & $10 \%$ \\
\hline Square & 34 & 6 & $15 \%$ \\
\hline Trigonometric & 38 & 2 & $5 \%$ \\
\hline
\end{tabular}

Table 12. The above tabulated value represents overall FNR Measure with Tensorflow from a document of 60 samples.

\begin{tabular}{|l|l|l|l|}
\hline $\begin{array}{l}\text { 60 Number of } \\
\text { testing formulae }\end{array}$ & $\boldsymbol{n}(\boldsymbol{T p})$ & $\boldsymbol{n}(\boldsymbol{F n})$ & $\begin{array}{l}\text { False Negative } \\
\text { Rate }\end{array}$ \\
\hline Addition & 58 & 2 & $3.33 \%$ \\
\hline Combination & 60 & 0 & $0 \%$ \\
\hline Differentiation & 60 & 0 & $0 \%$ \\
\hline Exponential & 59 & 1 & $1.66 \%$ \\
\hline Factorial & 59 & 1 & $1.66 \%$ \\
\hline Integral & 58 & 2 & $3.33 \%$ \\
\hline Limit & 60 & 0 & $0 \%$ \\
\hline Permutation & 58 & 2 & $3.33 \%$ \\
\hline Sigma & 56 & 4 & $6.67 \%$ \\
\hline Square root & 58 & 2 & $3.33 \%$ \\
\hline Square & 57 & 3 & $5 \%$ \\
\hline Trigonometric & 59 & 1 & $1.66 \%$ \\
\hline
\end{tabular}

\subsection{False Positive Rate}

FPR is the number of Math formulae, those responding positive on the test, means the math formulae which are correctly retrieved from the test document which are available in the training document as shown in tables 1315. The value FNR is mainly dependent on false positives. The number unwanted formulae retried with Tensorflow is almost zero as the procedure of retrieval of math formulae mainly depends on the training data.

FPR-False Positive Rate $=n\left(F_{p}\right) / n\left(F_{p}\right)+n\left(T_{n}\right)$
Table 13. The above tabulated value represents overall FPR Measure with Tensorflow from a document of 20 samples.

\begin{tabular}{|l|l|l|l|}
\hline $\begin{array}{l}\text { 20 Number of } \\
\text { testing formulae }\end{array}$ & $\boldsymbol{n}(\boldsymbol{T n})$ & $\boldsymbol{n}(\boldsymbol{F p})$ & $\begin{array}{l}\text { False Positive } \\
\text { Rate }\end{array}$ \\
\hline Addition & 0 & 0 & $0 \%$ \\
\hline Combination & 0 & 0 & $0 \%$ \\
\hline Differentiation & 0 & 0 & $0 \%$ \\
\hline Exponential & 0 & 0 & $0 \%$ \\
\hline Factorial & 0 & 0 & $0 \%$ \\
\hline Integral & 0 & 0 & $0 \%$ \\
\hline Limit & 0 & 0 & $0 \%$ \\
\hline Permutation & 0 & 0 & $0 \%$ \\
\hline Sigma & 0 & 0 & $0 \%$ \\
\hline Square root & 0 & 0 & $0 \%$ \\
\hline Square & 0 & 0 & $0 \%$ \\
\hline Trigonometric & 0 & 0 & $0 \%$ \\
\hline
\end{tabular}

Table 14. The above tabulated value represents overall FPR Measure with Tensorflow from a document of 40 samples.

\begin{tabular}{|l|l|l|l|}
\hline $\begin{array}{l}40 \text { Number of } \\
\text { testing formulae }\end{array}$ & $\boldsymbol{n}(\boldsymbol{T} \boldsymbol{p})$ & $\boldsymbol{n}(\boldsymbol{F p})$ & $\begin{array}{l}\text { False } \\
\text { Discovery Rate }\end{array}$ \\
\hline Addition & 0 & 0 & $0 \%$ \\
\hline Combination & 0 & 0 & $0 \%$ \\
\hline Differentiation & 0 & 0 & $0 \%$ \\
\hline Exponential & 0 & 0 & $0 \%$ \\
\hline Factorial & 0 & 0 & $0 \%$ \\
\hline Integral & 0 & 0 & $0 \%$ \\
\hline Limit & 0 & 0 & $0 \%$ \\
\hline Permutation & 0 & 0 & $0 \%$ \\
\hline Sigma & 0 & 0 & $0 \%$ \\
\hline Square root & 0 & 0 & $0 \%$ \\
\hline Square & 0 & 0 & $0 \%$ \\
\hline Trigonometric & 0 & 0 & $0 \%$ \\
\hline
\end{tabular}

Table 15. The above tabulated value represents overall FPR Measure with Tensorflow from a document of 60 samples.

\begin{tabular}{|l|l|l|l|}
\hline $\begin{array}{l}60 \text { Number of } \\
\text { testing formulae }\end{array}$ & $\boldsymbol{n}(\boldsymbol{T n})$ & $\boldsymbol{n}(\boldsymbol{F p})$ & $\begin{array}{l}\text { False Positive } \\
\text { Rate }\end{array}$ \\
\hline Addition & 0 & 0 & $0 \%$ \\
\hline Combination & 0 & 0 & $0 \%$ \\
\hline Differentiation & 0 & 0 & $0 \%$ \\
\hline Exponential & 0 & 0 & $0 \%$ \\
\hline Factorial & 0 & 0 & $0 \%$ \\
\hline Integral & 0 & 0 & $0 \%$ \\
\hline Limit & 0 & 0 & $0 \%$ \\
\hline Permutation & 0 & 0 & $0 \%$ \\
\hline Sigma & 0 & 0 & $0 \%$ \\
\hline Square root & 0 & 0 & $0 \%$ \\
\hline Square & 0 & 0 & $0 \%$ \\
\hline Trigonometric & 0 & 0 & $0 \%$ \\
\hline
\end{tabular}

\subsection{False Discovery Rate}


FDR is a much unfussy consideration. It is a ratio between the number of unwanted math formulae retrievals in a text document divided by total number of retrievals after comparison with training document and are accessible in tables 16-18. The value of FDR for different range of samples is $0 \%$ means no unwanted formulae are retrieved with proposed approach.

$$
\text { FDR - False Discovery Rate }=n\left(F_{p}\right) / n\left(F_{p}\right)+n\left(T_{p}\right)
$$

Table 16. The above tabulated value represents overall FDR Measure with Tensorflow from a document of 20 samples.

\begin{tabular}{|l|l|l|l|}
\hline $\begin{array}{l}\text { 20 Number of } \\
\text { testing formulae }\end{array}$ & $\boldsymbol{n}(\boldsymbol{T p})$ & $\boldsymbol{n}(\boldsymbol{F p})$ & $\begin{array}{l}\text { False } \\
\text { Discovery Rate }\end{array}$ \\
\hline Addition & 19 & 0 & $0 \%$ \\
\hline Combination & 19 & 0 & $0 \%$ \\
\hline Differentiation & 20 & 0 & $0 \%$ \\
\hline Exponential & 18 & 0 & $0 \%$ \\
\hline Factorial & 19 & 0 & $0 \%$ \\
\hline Integral & 19 & 0 & $0 \%$ \\
\hline Limit & 20 & 0 & $0 \%$ \\
\hline Permutation & 18 & 0 & $0 \%$ \\
\hline Sigma & 16 & 0 & $0 \%$ \\
\hline Square root & 16 & 0 & $0 \%$ \\
\hline Square & 16 & 0 & $0 \%$ \\
\hline Trigonometric & 19 & 0 & $0 \%$ \\
\hline
\end{tabular}

Table 17. The above tabulated value represents overall FDR Measure with Tensorflow from a document of 40 samples.

\begin{tabular}{|l|l|l|l|}
\hline $\begin{array}{l}\text { 40 Number of } \\
\text { testing formulae }\end{array}$ & $\boldsymbol{n ( T p )}$ & $\boldsymbol{n}(\boldsymbol{F p})$ & $\begin{array}{l}\text { False } \\
\text { Discovery Rate }\end{array}$ \\
\hline Addition & 38 & 0 & $0 \%$ \\
\hline Combination & 39 & 0 & $0 \%$ \\
\hline Differentiation & 40 & 0 & $0 \%$ \\
\hline Exponential & 38 & 0 & $0 \%$ \\
\hline Factorial & 39 & 0 & $0 \%$ \\
\hline Integral & 39 & 0 & $0 \%$ \\
\hline Limit & 40 & 0 & $0 \%$ \\
\hline Permutation & 36 & 0 & $0 \%$ \\
\hline Sigma & 34 & 0 & $0 \%$ \\
\hline Square root & 36 & 0 & $0 \%$ \\
\hline Square & 34 & 0 & $0 \%$ \\
\hline Trigonometric & 38 & 0 & $0 \%$ \\
\hline
\end{tabular}

Table 18. The above tabulated value represents overall FDR Measure with Tensorflow from a document of 60 samples.

\begin{tabular}{|l|l|l|l|}
\hline $\begin{array}{l}60 \text { Number of } \\
\text { testing formulae }\end{array}$ & $\boldsymbol{n ( T \boldsymbol { p } )}$ & $\boldsymbol{n}(\boldsymbol{F p})$ & $\begin{array}{l}\text { False } \\
\text { Discovery Rate }\end{array}$ \\
\hline Addition & 58 & 0 & $0 \%$ \\
\hline Combination & 60 & 0 & $0 \%$ \\
\hline Differentiation & 60 & 0 & $0 \%$ \\
\hline Exponential & 59 & 0 & $0 \%$ \\
\hline Factorial & 59 & 0 & $0 \%$ \\
\hline Integral & 58 & 0 & $0 \%$ \\
\hline Limit & 60 & 0 & $0 \%$ \\
\hline
\end{tabular}

\begin{tabular}{|l|l|l|l|}
\hline Permutation & 58 & 0 & $0 \%$ \\
\hline Sigma & 56 & 0 & $0 \%$ \\
\hline Square root & 58 & 0 & $0 \%$ \\
\hline Square & 57 & 0 & $0 \%$ \\
\hline Trigonometric & 59 & 0 & $0 \%$ \\
\hline
\end{tabular}

\subsection{Accuracy}

The accuracy of a test is its ability to categorize the retrieval of not needed and required math formulae acceptably. The accuracy can be calculated with the quantity of true positive and true negative in all assessed cases as shown in tables 19-21. The Accuracy of retrieval of math formulae increases with increase in number of samples.

Accuracy ACC $=n\left(T_{p}\right)+n\left(T_{n}\right) / n\left(T_{p}\right)+n\left(T_{n}\right)+n\left(F_{p}\right)+n\left(F_{n}\right)$ (6)

Table 19. The above tabulated value represents overall Accuracy Measure with Tensorflow from a document of 20 samples.

\begin{tabular}{|l|l|l|l|l|l|}
\hline $\begin{array}{l}20 \text { Number } \\
\text { of testing } \\
\text { formulae }\end{array}$ & $\boldsymbol{n}(\boldsymbol{T p})$ & $\boldsymbol{n}(\boldsymbol{F p})$ & $\boldsymbol{n}(\boldsymbol{T n})$ & $\boldsymbol{n}(\boldsymbol{F n})$ & Accuracy \\
\hline Addition & 19 & 0 & 0 & 1 & $95 \%$ \\
\hline Combination & 19 & 0 & 0 & 1 & $95 \%$ \\
\hline Differentiation & 20 & 0 & 0 & 0 & $100 \%$ \\
\hline Exponential & 18 & 0 & 0 & 2 & $90 \%$ \\
\hline Factorial & 19 & 0 & 0 & 1 & $95 \%$ \\
\hline Integral & 19 & 0 & 0 & 1 & $95 \%$ \\
\hline Limit & 20 & 0 & 0 & 0 & $100 \%$ \\
\hline Permutation & 18 & 0 & 0 & 2 & $90 \%$ \\
\hline Sigma & 16 & 0 & 0 & 4 & $80 \%$ \\
\hline Square root & 16 & 0 & 0 & 4 & $80 \%$ \\
\hline Square & 16 & 0 & 0 & 4 & $80 \%$ \\
\hline Trigonometric & 19 & 0 & 0 & 1 & $95 \%$ \\
\hline
\end{tabular}

Table 20. The above tabulated value represents overall Accuracy Measure with Tensorflow from a document of 40 samples.

\begin{tabular}{|l|l|l|l|l|l|}
\hline $\begin{array}{l}40 \text { Number } \\
\text { of testing } \\
\text { formulae }\end{array}$ & $\boldsymbol{n}(\boldsymbol{T p})$ & $\boldsymbol{n}(\boldsymbol{F p})$ & $\boldsymbol{n}(\boldsymbol{T n})$ & $\boldsymbol{n}(\boldsymbol{F n})$ & $\begin{array}{l}\text { Accurac } \\
\mathrm{y}\end{array}$ \\
\hline Addition & 38 & 0 & 0 & 2 & $95 \%$ \\
\hline Combination & 39 & 0 & 0 & 1 & $97.5 \%$ \\
\hline $\begin{array}{l}\text { Differentiatio } \\
\mathrm{n}\end{array}$ & 40 & 0 & 0 & 0 & $100 \%$ \\
\hline Exponential & 38 & 0 & 0 & 2 & $95 \%$ \\
\hline Factorial & 39 & 0 & 0 & 1 & $97.5 \%$ \\
\hline Integral & 39 & 0 & 0 & 1 & $97.5 \%$ \\
\hline Limit & 40 & 0 & 0 & 0 & $100 \%$ \\
\hline Permutation & 36 & 0 & 0 & 4 & $90 \%$ \\
\hline Sigma & 34 & 0 & 0 & 6 & $85 \%$ \\
\hline
\end{tabular}




\begin{tabular}{|l|l|l|l|l|l|}
\hline Square root & 36 & 0 & 0 & 4 & $90 \%$ \\
\hline Square & 34 & 0 & 0 & 6 & $85 \%$ \\
\hline $\begin{array}{l}\text { Trigonometri } \\
\mathrm{c}\end{array}$ & 38 & 0 & 0 & 2 & $95 \%$ \\
\hline
\end{tabular}

Table 21. The above tabulated value represents overall Accuracy Measure with Tensorflow from a document of 60 samples.

\begin{tabular}{|l|l|l|l|l|l|}
\hline $\begin{array}{l}60 \text { Number } \\
\text { of testing } \\
\text { formulae }\end{array}$ & $\boldsymbol{n}(\boldsymbol{T p})$ & $\boldsymbol{n}(\boldsymbol{F p})$ & $\boldsymbol{n}(\boldsymbol{T n})$ & $\boldsymbol{n}(\boldsymbol{F n})$ & Accuracy \\
\hline Addition & 58 & 0 & 0 & 2 & $96.7 \%$ \\
\hline Combination & 60 & 0 & 0 & 0 & $100 \%$ \\
\hline Differentiation & 60 & 0 & 0 & 0 & $100 \%$ \\
\hline Exponential & 59 & 0 & 0 & 1 & $98.3 \%$ \\
\hline Factorial & 59 & 0 & 0 & 1 & $98.3 \%$ \\
\hline Integral & 58 & 0 & 0 & 2 & $96.7 \%$ \\
\hline Limit & 60 & 0 & 0 & 0 & $100 \%$ \\
\hline Permutation & 58 & 0 & 0 & 2 & $96.7 \%$ \\
\hline Sigma & 56 & 0 & 0 & 4 & $93.3 \%$ \\
\hline Square root & 58 & 0 & 0 & 2 & $98.3 \%$ \\
\hline Square & 57 & 0 & 0 & 3 & $95 \%$ \\
\hline Trigonometric & 59 & 0 & 0 & 1 & $98.3 \%$ \\
\hline
\end{tabular}

\subsection{Positive Predictive Value}

Positive predictive value (PPV) is a measure of significant occurrences amid the retrieved occurrences it is also known as precision. The PPV value with the proposed approach is $100 \%$ for different number of samples with different dominating types of formulae as shown in tables 22-24.

Positive Predictive value $(P P V)=n\left(T_{p}\right) / n\left(T_{p}\right)+n\left(F_{p}\right)$

Table 22. The above tabulated value represents overall PPV Measure with Tensorflow from a document of 60 samples.

\begin{tabular}{|l|l|l|l|}
\hline $\begin{array}{l}\text { 20 Number of } \\
\text { testing formulae }\end{array}$ & $\boldsymbol{n}(\boldsymbol{T p})$ & $\boldsymbol{n}(\boldsymbol{F p})$ & $\begin{array}{l}\text { False } \\
\text { Discovery Rate }\end{array}$ \\
\hline Addition & 19 & 0 & $100 \%$ \\
\hline Combination & 19 & 0 & $100 \%$ \\
\hline Differentiation & 20 & 0 & $100 \%$ \\
\hline Exponential & 18 & 0 & $100 \%$ \\
\hline Factorial & 19 & 0 & $100 \%$ \\
\hline Integral & 19 & 0 & $100 \%$ \\
\hline Limit & 20 & 0 & $100 \%$ \\
\hline Permutation & 18 & 0 & $100 \%$ \\
\hline Sigma & 16 & 0 & $100 \%$ \\
\hline Square root & 16 & 0 & $100 \%$ \\
\hline Square & 16 & 0 & $100 \%$ \\
\hline Trigonometric & 19 & 0 & $100 \%$ \\
\hline & & &
\end{tabular}

Table 23. The above tabulated value represents overall PPV Measure with Tensorflow from a document of 60 samples.

\begin{tabular}{|l|l|l|l|}
\hline $\begin{array}{l}40 \text { Number of } \\
\text { testing formulae }\end{array}$ & $\boldsymbol{n}(\boldsymbol{T p})$ & $\boldsymbol{n}(\boldsymbol{F p})$ & $\begin{array}{l}\text { False Discovery } \\
\text { Rate }\end{array}$ \\
\hline Addition & 38 & 0 & $100 \%$ \\
\hline Combination & 39 & 0 & $100 \%$ \\
\hline Differentiation & 40 & 0 & $100 \%$ \\
\hline Exponential & 38 & 0 & $100 \%$ \\
\hline Factorial & 39 & 0 & $100 \%$ \\
\hline Integral & 39 & 0 & $100 \%$ \\
\hline Limit & 40 & 0 & $100 \%$ \\
\hline Permutation & 36 & 0 & $100 \%$ \\
\hline Sigma & 34 & 0 & $100 \%$ \\
\hline Square root & 36 & 0 & $100 \%$ \\
\hline Square & 34 & 0 & $100 \%$ \\
\hline Trigonometric & 38 & 0 & $100 \%$ \\
\hline
\end{tabular}

Table 24. The above tabulated value represents overall PPV Measure with Tensorflow from a document of 60 samples.

\begin{tabular}{|l|l|l|l|}
\hline $\begin{array}{l}\text { 60 Number of } \\
\text { testing formulae }\end{array}$ & $\boldsymbol{n}(\boldsymbol{T p})$ & $\boldsymbol{n}(\boldsymbol{F p})$ & $\begin{array}{l}\text { False } \\
\text { Discovery Rate }\end{array}$ \\
\hline Addition & 58 & 0 & $100 \%$ \\
\hline Combination & 60 & 0 & $100 \%$ \\
\hline Differentiation & 60 & 0 & $100 \%$ \\
\hline Exponential & 59 & 0 & $100 \%$ \\
\hline Factorial & 59 & 0 & $100 \%$ \\
\hline Integral & 58 & 0 & $100 \%$ \\
\hline Limit & 60 & 0 & $100 \%$ \\
\hline Permutation & 58 & 0 & $100 \%$ \\
\hline Sigma & 56 & 0 & $100 \%$ \\
\hline Square root & 58 & 0 & $100 \%$ \\
\hline Square & 57 & 0 & $100 \%$ \\
\hline Trigonometric & 59 & 0 & $100 \%$ \\
\hline
\end{tabular}

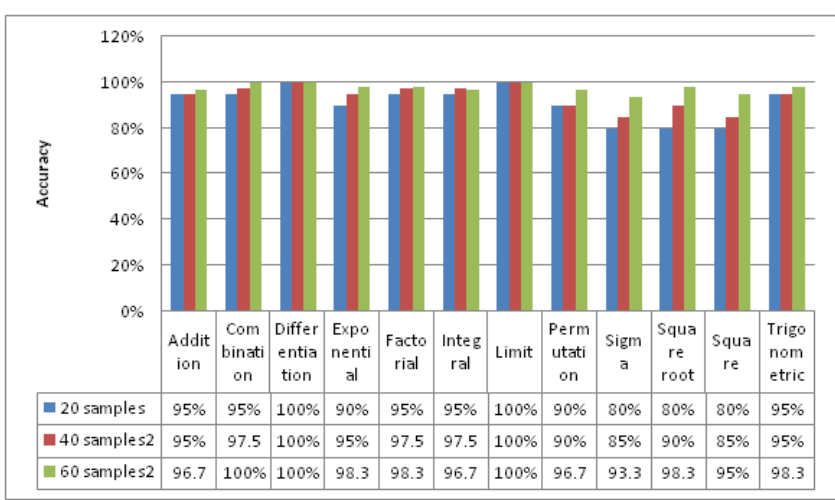

Figure 2. Overall Accuracy Measure with Tensorflow from a document. 


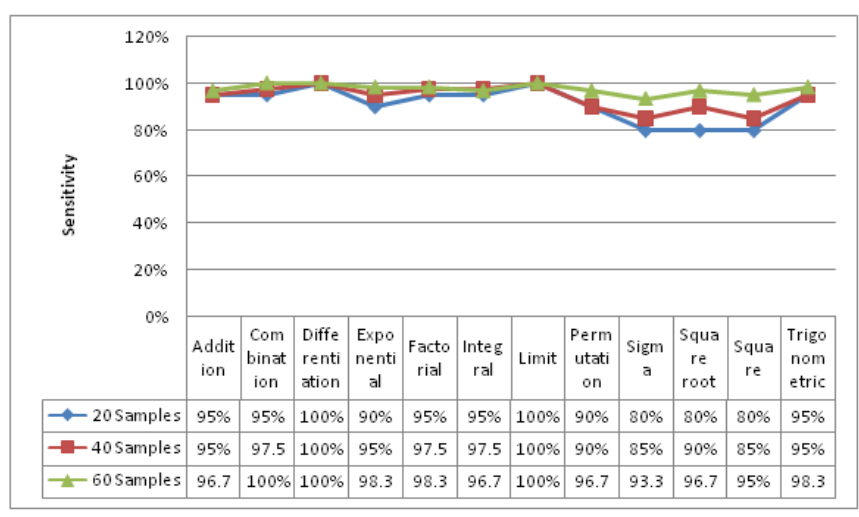

Figure 3. Overall Sensitivity Measure with Tensorflow from a document.

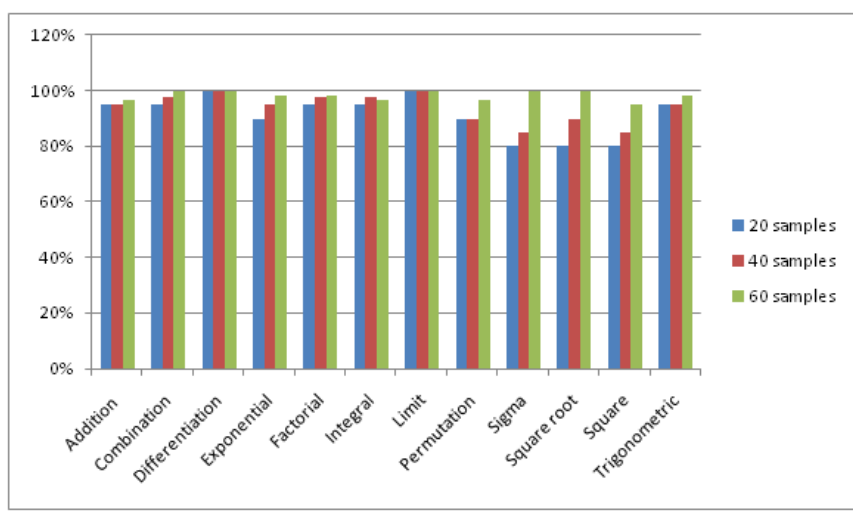

Figure 4. Efficiency Measure with Tensorflow from the document.

\section{Conclusion}

In this article an approach which retrieves mathematical formulae was projected. The efficiency of the wished-for procedure presented in terms of time analysis and accuracy. The proposed Tensorflow based math classification retrieves all the math formulae that are matched with data in the training document. The efficiency of proposed method is evaluated in terms of metrics like Sensitivity, Efficiency, Accuracy, PPV FDR, FPR and FNR. As more number of matched formulae and no unwanted formulae are retrieved with Tensorflow based math classification it out performs in terms of efficiency. The efficiency increases with increase in the number of math formulae in training document. The proposed method with Tensolflow produces best results in terms of Sensitivity, Specificity, PPV, False Positive Rate and False Negative Rate.

\section{References}

[1] K Ma, S Cheung Hui and K Chang, "Feature Extraction and Clustering-based Retrieval for Mathematical Formulas", The 2nd International Conference on Software Engineering and Data Mining, pp. 372-377, 2010.

[2]S Harshanath Samarasinghe and S Cheung Hui, "Mathematical Document Retrieval for Problem Solving", International Conference on Computer Engineering and Technology, pp.583$587,2009$.

[3] J Misutka and L Galambos, "Mathematical Extension of Full Text Search Engine Indexer", Proc. 3rd International Conference on Information and Communication Technologies: From Theory to Applications (ICTTA 08), pp. 1-6April 2008.

[4] Web Link to TensorFlow, Last accessed on $15^{\text {th }}$ may 2019 https://sourcedexter.com/tensorflow-text-classification-python/. [5] B R Miller and A Youssef, "Technical Aspects of the Digital Library of Mathematical Functions", in Annals of Mathematics and Artificial Intelligence, Springer Netherlands, pp. 121-136, 2003.

[6] H Zhang, T B and M S Lin, "An Evolutionary K means Algorithm for Clustering Time Series Data", Proc. International Conference on Machine Learning and Cybernetics, pp. 12821287, 2004.

[7] R Munavalli and M R MathFind, "A Math-aware Search Engine", Proc. Annual International ACM SIGIR Conference on Research and development in information retrieval, pp.735-735, 2006.

[8] M Kohlhase, "Markup for Mathematical Knowledge", An Open Markup format for Mathematical Documents, Ver. 1.2, Lecture Notes in Computer Science, pp. 13-23, Springer Berlin,2006.

[9] G Appa Rao, K Venkata Rao, P V G D Prasad Reddy and T Lava Kumar, "An Efficient Procedure for Characteristic mining of Mathematical Formulas from Document", International Journal of Engineering Science and Technology (IJEST), Vol. 10 No.03, pp152-157, Mar 2018.

[10] G Appa Rao, G Srinivas, K Venkata Rao and P V G D Prasad Reddy," Characteristic mining of Mathematical Formulas from Document - A Comparative Study on Sequence Matcher and Levenshtein Distance procedure", International Journal of 
Computer Sciences and Engineering, Volume-6, Issue-4, pp 400-403, Apr 2018.

[11] G AppaRao, G Srinivas, K Venkata Rao and P V G D Prasad Reddy, "a partial ratio and ratio based fuzzy-wuzzy procedure for characteristic mining of mathematical formulas from documents", IJSC- ICTACT Journal on Soft Computing, Vol 8, Issue 4, pp 1728-1732, July 2018.

[12] M Peng, D Chen, Q Xie, Y Zhang, H Wang, G Hu, W Gao, Y Zhang, " Topic-Net Conversation Model ", International Conference on Web Information Systems Engineering, pp.483496, 2018.

[13] M Peng, Q Xie, Y Zhang, H Wang, X Zhang, J Huang, G Tian, “Neural Sparse Topical Coding ”, Proceedings of the $56^{\text {th }}$ Annual Meeting of the Association for Computational Linguistics (volume 1: Long Papers), pp.2332-2340, 2018.

[14] M Peng, Q Xie, H Wang, Y Zhang, G Tian, ” Bayesian Sparse Topical Coding ", IEEE Transactions on Knowledge and Data Engineering, pp.1080-1093, 2018.

[15] H Wang and Y Zhang, "An Efficient Framework for The Analysis of Big Brain Signals Data", Australasian Database Conference, pp.199-207, 2018.

[16] M Peng, H Shi, Q Xie, Y Zhang, H Wang, Z Li, J Yong, "Block Bayesian Sparse Topical Coding", 2018 IEEE 22 ${ }^{\text {nd }}$ International Conference on Computer Supported Cooperative Work in Design((CSCWD)), pp.271-276, Nanjing, 2018.

[17] M Peng, J Zhu, H Wang, X Li, Y Zhang, X Zhang, G Tian, "Mining event-oriented topics in microblog steam with unsupervised multi-view hierarchical embedding", ACM Transactions on Knowledge Discovery from Data(TKDD) 12(3), Article No.38, 2018. 